\title{
Induced fibrillation is equally effective as crystalloid cardioplegia in the protection of fetal myocardial function
}

\author{
Sunil P. Malhotra, MD \\ Stephan Thelitz, MD \\ R. Kirk Riemer, PhD \\ V. Mohan Reddy, MD \\ Sam Suleman, BS \\ Frank L. Hanley, MD
}

From the Department of Cardiothoracic Surgery, Stanford University School of Medicine, Stanford, Calif.

Read at the Eighty-second Annual Meeting of The American Association for Thoracic Surgery, Washington, DC, May 5-8, 2002.

Dr Malhotra is a recipient of the National Research Service Award (F32 HL 1033901) from the National Heart, Lung, and Blood Institute of the National Institutes of Health.

Received for publication May 29, 2002; revisions requested July 8, 2002; revisions received Oct 4, 2002; accepted for publication Oct 17, 2002.

Address for reprints: Sunil P. Malhotra, MD, New York University School of Medicine, Department of Surgery, 530 First Ave, NB-15N1, New York, NY 10016 (Email: spmalhotra@yahoo.com).

J Thorac Cardiovasc Surg 2003;125: 1276-82

Copyright $\odot 2003$ by The American Association for Thoracic Surgery

$0022-5223 / 2003 \$ 30.00+0$

doi:10.1016/S0022-5223(02)73245-5
Background: Fetal cardiac intervention represents a potential advance in the treatment of congenital cardiac lesions that increase in complexity during development. Prenatal repair of a primary defect might prevent pathologic blood-flow patterns that can result in hypoplasia of a cardiac chamber or great vessel. However, strategies to optimize fetal myocardial protection have not been studied. A biventricular working fetal heart preparation was used to evaluate the cardioprotective properties of induced fibrillation and crystalloid cardioplegia.

Methods: Hearts from 16 fetal lambs at 115 to 125 days' gestation were harvested and perfused with Krebs-Henseleit solution. The descending aorta was ligated distal to the ductal insertion and the branch pulmonary arteries were ligated to simulate the parallel circulation of the fetus. Hearts were arrested with normothermic fibrillation $(n=8)$ or hypothermic crystalloid cardioplegia $(n=8)$ before reperfusion with Krebs-Henseleit solution. Baseline and postarrest myocardial function measurements were obtained from analysis of pressure-dimension relationships.

Results: Fibrillatory and cardioplegic arrest were equally effective at preserving postarrest systolic function (left ventricle, $70 \% \pm 5 \%$ vs $68 \% \pm 15 \%, P=.52$; right ventricle, $68 \% \pm 4.5 \%$ vs $65 \% \pm 4.5 \%, P=.26$ ) and preventing increased diastolic stiffness (left ventricle, $32 \% \pm 5.3 \%$ vs $38 \% \pm 11 \%, P=.24$; right ventricle, $25 \%$ $\pm 3.3 \%$ vs $27 \% \pm 2.1 \%, P=.46)$. Myocardial water content was unchanged in hearts arrested with fibrillation and cardioplegia $(84 \% \pm 1.5 \%$ vs $83.7 \% \pm 0.9 \%$, $P=.71)$.

Conclusions: Normothermic fibrillation and hypothermic crystalloid cardioplegia provide equal protection of the fetal myocardium. In the setting of diminished fetal myocardial reserve and because of the limited ability to manipulate the surrounding temperature in the fetus, normothermic fibrillation may be preferable for in utero repairs of selected congenital heart defects.

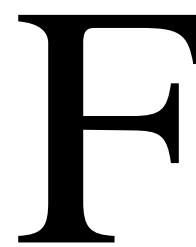
etal cardiac surgery offers the promise of in utero repair of complex congenital defects that evolve during development. A primary lesion, such as valvular stenosis, might develop during primary morphogenesis, altering blood flow. The resulting pathologic blood-flow pattern in the developing heart induces hypoplasia of a heart chamber or great vessel. ${ }^{1}$ It is this secondary lesion that causes hemodynamic instability in the neonatal period.

Targeting the primary defect during the fetal period might restore normal intracardiac flow patterns. Repair of the primary lesion should be relatively uncomplicated, allowing the fetal heart to rapidly recover and prepare for the transition to 


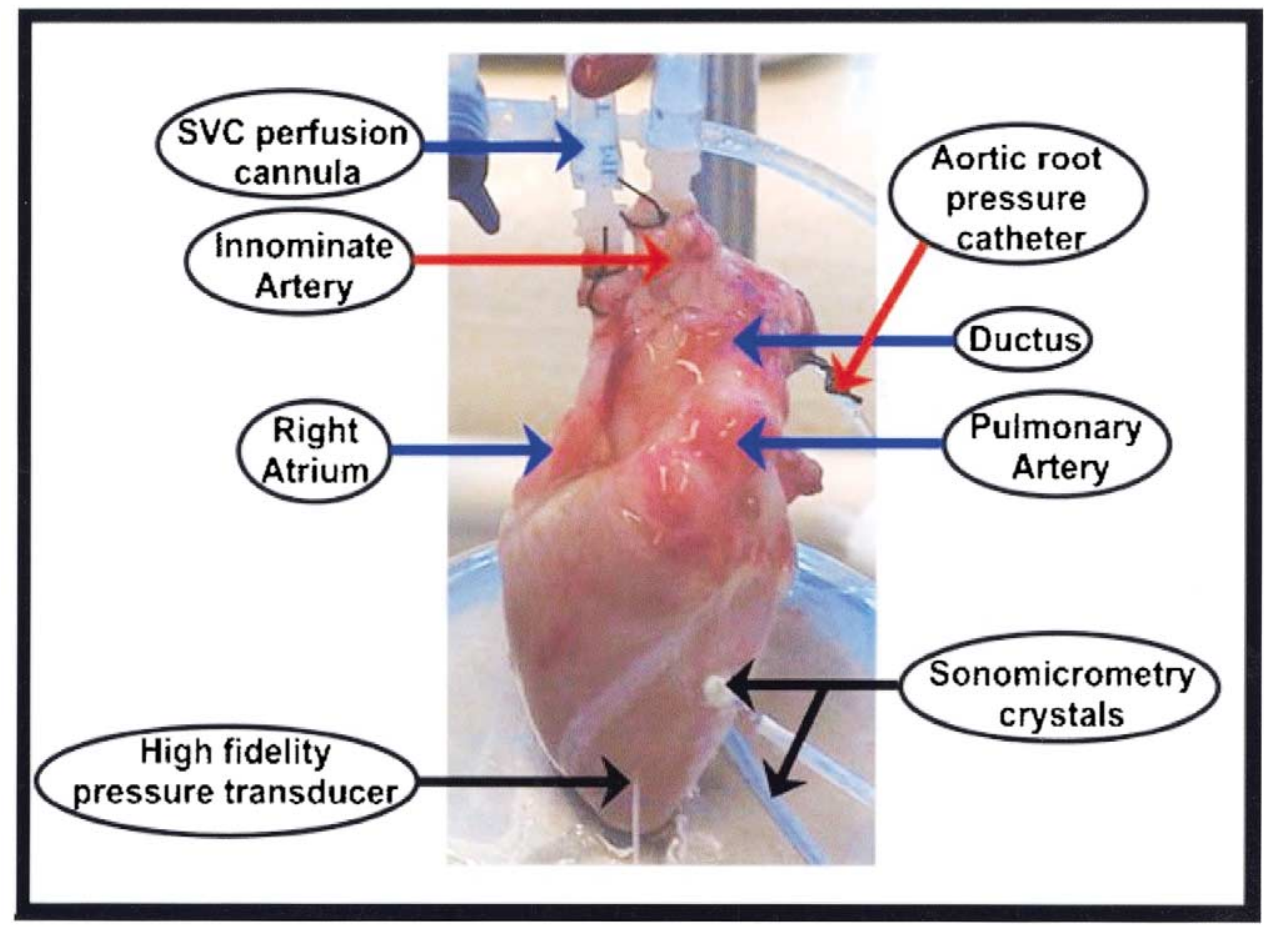

Figure 1. Isolated biventricular fetal heart preparation. Langendorff mode involves perfusion directly through the aortic root. In the working heart mode perfusion is through the SVC cannula.

postnatal circulation. Fortunately, recent advances in cardiovascular imaging have increased the likelihood of successful fetal cardiac interventions. Currently, fetal echocardiography can be used to accurately diagnose certain congenital heart defects as early as 10 to 12 weeks of gestation. $^{2}$

Numerous studies have demonstrated that fetal hemodynamic stability after cardiac bypass is dependent on the degree of stress to the fetoplacental unit. ${ }^{3,4}$ To that end, limiting ischemic myocardial damage by optimizing myocardial protection is essential to achieving a successful outcome of fetal cardiac surgery. Appropriate cardioprotection strategies must reflect the unique aspects of fetal myocardial physiology, including the impaired Frank-Starling mechanism and diastolic compliance of the fetal myocardium. $^{5}$

Because prenatal repairs will, in certain circumstances, require short periods of arrest, fibrillatory arrest should be a safe approach for operations on the fetal heart. Studies in fetal lambs found that regional myocardial blood flows were preserved during cardiac bypass with normothermic fibrillatory arrest. ${ }^{6,7}$ Jessen and colleagues ${ }^{8}$ found that fibrillation caused a $15 \%$ increase in neonatal myocardial oxygen consumption compared with $50 \%$ in studies on fibrillating adult hearts. This observed difference may reflect the immaturity of the myocardial contractile elements. Fetal myocardium might exhibit further attenuation of increased myocardial oxygen consumption after fibrillatory arrest because of its inability to mobilize calcium stores. In this study the efficacy of fibrillatory and cardioplegic arrest in the preservation of fetal myocardial function was examined by using an isolated, biventricular, fetal working heart preparation that closely simulates fetal cardiovascular physiology.

\section{Methods}

\section{Experimental Preparation}

Hearts from 16 fetal lambs (119 \pm 3.1 days' gestation, $2.5 \pm 0.4$ $\mathrm{kg}$ ) were harvested and mounted on a controlled perfusion apparatus (Figure 1). Pregnant ewes were sedated with intramuscular ketamine $(10 \mathrm{mg} / \mathrm{kg})$. Ewes were then anesthetized with inhaled isoflurane (2\%), intubated, and mechanically ventilated. After maternal laparotomy and uterotomy, the fetal chest was exposed. A midline sternotomy was performed, and the great vessels were identified. A 1500-unit dose of heparin was administered to account for both the fetus and placenta. The descending aorta was transected distal to the ductal insertion. The heart was removed after transection of the inferior vena cava, the superior vena cava (SVC), and the innominate artery. The heart was excised along with a cuff of lung tissue to facilitate identification of the pulmonary arteries and veins during mounting. The heart was placed in Krebs-Henseleit bicarbonate buffer equilibrated with $95 \% \mathrm{CO}_{2}$ and 5\% $\mathrm{O}_{2}$ and transported to the water-jacketed perfusion apparatus (Radnotti Glass Technology, Inc). The perfusate was main- 
tained at a $\mathrm{Po}_{2}$ of $30 \mathrm{~mm} \mathrm{Hg}$ by using blood gas measurements to simulate the hypoxic conditions of the fetal heart.

During continuous aortic perfusion with Krebs-Henseleit at $37^{\circ} \mathrm{C}$, the innominate artery was cannulated with a flexible plastic cannula. A similar perfusion cannula was placed through the SVC into the right atrium (RA). A pressure catheter was placed through the transected descending aorta into the aortic root. The aorta was then ligated distal to the ductus arteriosus. The branch pulmonary arteries were ligated with metal clips to approximate the high fetal pulmonary vascular resistance. A second pressure catheter was secured in the RA through the inferior vena cava. Sonomicrometry crystals (Sonometrics Corp) were fixed to the epicardium with cyanoacrylic glue to measure changes in ventricular dimension. Two crystals were placed at opposite ends of the short axis of the left ventricle (LV), and an additional 2 crystals were similarly placed on the short axis of the right ventricle (RV). High-fidelity transducer-tipped catheters (Millar Instruments) were inserted into both the LV and RV.

All animals received humane care in compliance with the "Principles of Laboratory Animal Care" formulated by the National Society of Medical Research and the "Guide for the Care and Use of Laboratory Animals" prepared by the Institute of Laboratory Animal Resources and published by the National Institutes of Health. The protocol was approved by the Committee on Animal Research at the University of California, San Francisco.

\section{Data Acquisition}

Pressure-dimension (PD) loops were generated by using the Sonoview software package (Sonometrics Corp) from data acquired from the crystals and the transducer-tipped catheters. Inflow occlusion was used to generate descending PD loops. Heart rate, aortic root pressure, and RA pressure were measured with Transpal pressure transducers (Abbot Labs). Myocardial temperature was measured with an electronic temperature probe (YSI).

\section{Baseline Measurements}

After 10 minutes of direct aortic perfusion (Langendorff mode), perfusion through the SVC into the RA was initiated (working heart mode). During the working heart mode, both ventricles eject against a constant afterload created by $10 \mathrm{~cm}$ of 3/8-inch Tygon polyvinyl tubing attached to the aortic outflow. Baseline hemodynamic measurements were taken after 15 minutes in the working heart mode.

\section{Induction of Cardiac Arrest}

Cardioplegic arrest $(n=8)$ was induced and maintained for 30 minutes by means of a single infusion of Plegisol (Abbot Labs) crystalloid cardioplegic solution at $6^{\circ} \mathrm{C}(20 \mathrm{~mL} / \mathrm{kg})$ into the aortic root at a pressure of $20 \mathrm{~mm} \mathrm{Hg}$. Fibrillatory arrest $(\mathrm{n}=8)$ was accomplished with a $1.5-\mathrm{V}$ 60-cycle/s AC fibrillator (Department of Bioengineering, University of California, San Francisco). Hearts were continuously fibrillated for 30 minutes. A perfusion pressure of $40 \mathrm{~mm} \mathrm{Hg}$ was maintained through the aortic root during the fibrillation period. At the end of the arrest period, the fibrillation current was discontinued, and hearts were defibrillated with $5 \mathrm{~J}$ to restore sinus rhythm.

\section{Reperfusion}

In the cardioplegia group aortic perfusion was restarted through the aortic root after 30 minutes of cardiac arrest and was continued for 10 minutes after mechanical activity returned. In the fibrillation group the Langendorff mode was continued for 10 minutes once sinus rhythm returned. In both groups the working mode was then resumed, and postarrest measurements were taken after 15 minutes.

\section{Calculations of Myocardial Function}

Systolic, diastolic, and overall contractile function was calculated from PD loops by using the Cardiosoft software package (Sonometrics Corp). End-systolic elastance (ESE) is an index of systolic contractility calculated from the slope of the regression line that fits the end-systolic points on the PD loops. Diastolic stiffness is the end-diastolic PD relationship obtained from the exponential fit of the end-diastolic PD points on the PD loop. Preload recruitable stroke work (PRSW) is a preload-independent index of overall myocardial function. PRSW represents the linear relationship between stroke work and end-diastolic volume.

\section{Myocardial Water Determinations}

On completion of the study, the left ventricular free wall was excised and placed in a preweighed dish, and the wet weight was obtained. The sample was heated at $80^{\circ} \mathrm{C}$ until the weight remained constant to obtain the dry weight.

\section{Statistical Analysis}

All data are reported as means \pm SD. Analysis of statistical significance was performed by the Student $t$ test. All tests were paired and 2-tailed. Significance was established at the $95 \%$ confidence level. All values are expressed as mean $\pm \mathrm{SD}$. Analyses were performed with the Statview statistical package (SAS).

\section{Results}

Prearrest and postarrest hemodynamic data (heart rate, aortic pressure, right atrial pressure, and cardiac output) are presented in Table 1. These parameters are within the normal fetal physiologic range and are consistent between the 2 experimental groups.

There was no change in the prebypass and postbypass $\mathrm{X}$-axis intercept values $\left(\mathrm{V}_{0}\right)$ for elastance ( $\mathrm{LV}$ before bypass, $19.5 \pm 0.2 ; \mathrm{LV}$ after bypass, $19.2 \pm 0.1$; RV before bypass, $18.6 \pm 0.2 ; \mathrm{RV}$ after bypass, $18.8 \pm 0.1$ ) or PRSW (LV before bypass, $23.5 \pm 0.2 ; \mathrm{LV}$ after bypass, $23.3 \pm 0.1$; RV before bypass, $22.2 \pm 0.2$; RV after bypass, $22.4 \pm 0.1$ ) in the fibrillation or cardioplegia groups. Accordingly, ESE, diastolic stiffness, and PRSW are represented by the change of the slope after arrest or fibrillation.

\section{Recovery of Systolic Function}

Recovery of ESE is shown in Figure 2. Fibrillation and cardioplegia provided equal protection of systolic function. After cardiac arrest, the percentage recovery of left ventricular ESE was $70 \% \pm 5 \%$ in the fibrillation group and $68 \%$ $\pm 15 \%$ with cardioplegic arrest $(P=.52)$. Preservation of 


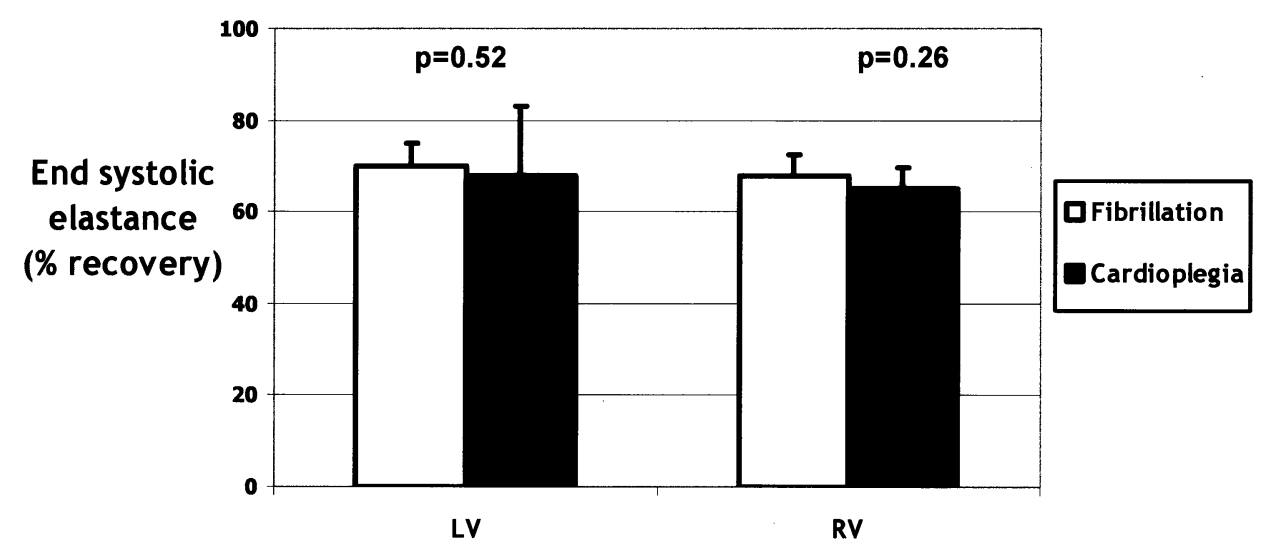

Figure 2. Ventricular systolic function measured on the basis of ESE. Percentage recovery represents the ratio of postarrest ESE to prearrest ESE. Fibrillation and cardioplegia provide equivalent protection of right and left ventricular systolic function.

right ventricular systolic function was also statistically equivalent with either technique, with a $68 \% \pm 4.5 \%$ recovery of elastance in fibrillating hearts and $65 \% \pm 4.5 \%$ recovery of baseline ESE with cardioplegic arrest $(P=.26)$.

\section{Protection From Diastolic Dysfunction}

Cardioplegic and fibrillatory arrest equally preserved biventricular diastolic compliance (Figure 3). Left ventricular diastolic stiffness increased $32 \% \pm 5.3 \%$ in the fibrillation arrest group and $38 \% \pm 11 \%$ in the cardioplegic arrest cohort $(P=.24)$. There was no statistically significant difference in increased postarrest right ventricular diastolic stiffness between the fibrillation and cardioplegia groups $(25 \% \pm 3.3 \%$ vs $27 \% \pm 2.1 \%$, respectively; $P=.46)$.

\section{Preservation of Overall Myocardial Function}

Figure 4 depicts the postarrest recovery of PRSW. Fibrillation and cardioplegia provided equal levels of protection of overall left ventricular myocardial function, with $77 \% \pm$ $3.6 \%$ and $75 \% \pm 13 \%$ recovery of PSRW, respectively $(P$ $=.82$ ). Recovery of right ventricular PRSW was $81 \% \pm$ $9.1 \%$ with fibrillatory arrest and $79 \% \pm 4.5 \%$ with cardioplegic arrest $(P=.69)$.

\section{Myocardial Edema}

There was no difference in postarrest myocardial edema after either fibrillation or cardioplegia. Water content was $83.7 \% \pm 0.9 \%$ and $84.0 \% \pm 1.5 \%$ after fibrillatory and cardioplegic arrest, respectively $(P=.71$, Figure 5$)$. In contrast, the baseline water content in age-matched control fetal hearts was $77.8 \% \pm 0.7 \%$.

\section{Discussion}

Methods need to be developed to safely protect the fetal myocardium during periods of cardiac intervention when
TABLE 1. Prearrest and postarrest hemodynamic data

\begin{tabular}{lcr}
\hline & Fibrillation & Cardioplegia \\
\hline Prearrest data & & \\
$\quad$ Heart rate & $141 \pm 14.1$ & $144.5 \pm 4.1$ \\
Mean aortic root pressure & $30.4 \pm 5.5$ & $32.8 \pm 3.2$ \\
Right atrial pressure & $5.8 \pm 1.7$ & $6.2 \pm 1.2$ \\
$\quad$ Cardiac output (mL/min) & $94.6 \pm 12.6$ & $95.5 \pm 7.6$ \\
Postarrest data & & \\
$\quad$ Heart rate & $142.7 \pm 11.3$ & $146.5 \pm 8.5$ \\
Mean aortic root pressure & $32.9 \pm 3.4$ & $33.5 \pm 6.4$ \\
Right atrial pressure & $6.0 \pm 0.8$ & $6.5 \pm 1.29$ \\
Cardiac output (mL/min) & $88.7 \pm 13.0$ & $90.3 \pm 18.4$ \\
\hline
\end{tabular}

the heart is not beating. Myocardial protection is critical for the fetus because of its limited cardiac reserve and increased sensitivity to ischemic stress. Even neonatal myocardial protection strategies may not be applicable to the developing heart because of the immaturity of the calcium regulatory apparatus in the fetal myocardium. The sarcoplasmic reticulum (SR) differs significantly in morphology and function in fetal myocytes. Myocardial SR content is markedly reduced in the fetal myocardium. ${ }^{9}$ Furthermore, fetal SR structure is immature because of decreased concentrations of calcium ATPase and calsequestran. ${ }^{10}$ As a result, regulation of myocardial calcium concentration is suboptimal in the fetus. These findings might explain the lack of inotropic reserve of the fetal ventricle. ${ }^{11}$

This model is designed to mimic in vivo fetal cardiac dynamics by including the ductus in the circuit and ligating the branch pulmonary arteries so that both ventricles are performing in parallel, as in fetal life. As with other isolated heart preparations, this model does not account for the effects of noncoronary collaterals or bronchial blood flow. Nonetheless, use of an isolated preparation to study fetal 


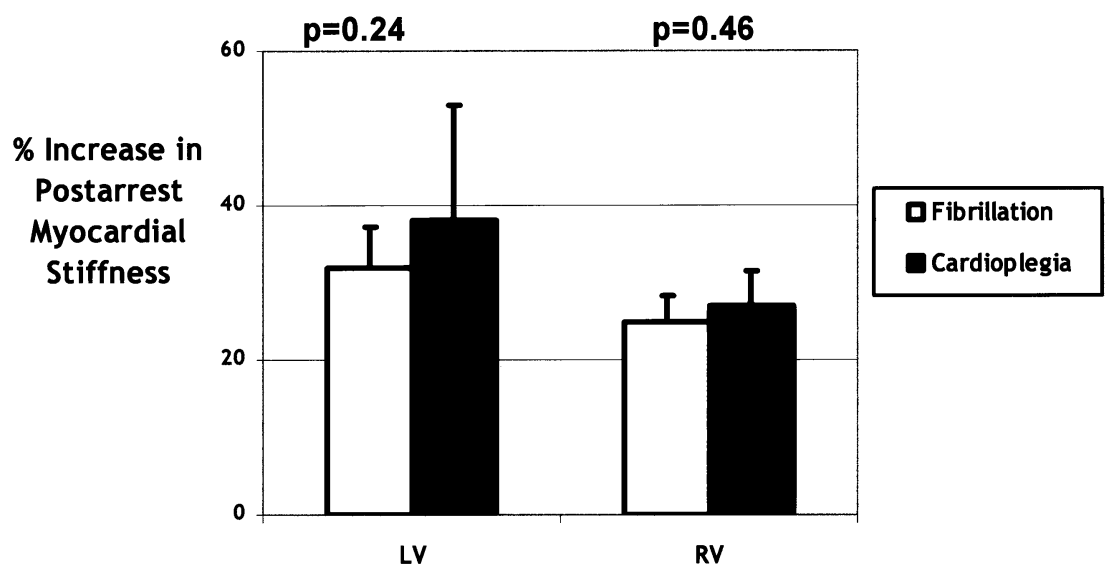

Figure 3. Ventricular diastolic stiffness expressed as a percentage of postarrest stiffness compared with prearrest values. Diastolic function of both ventricles is equally preserved after fibrillatory and cardioplegic arrest.

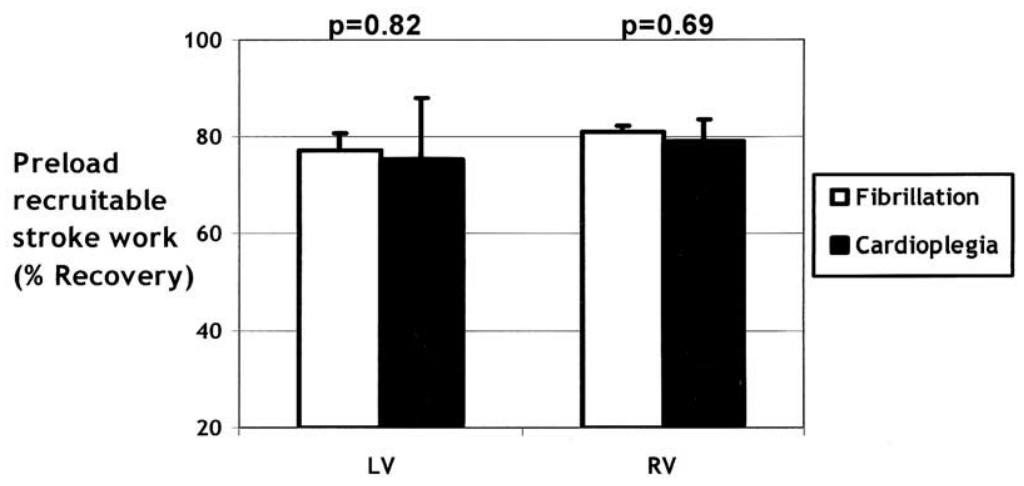

Figure 4. Recovery of myocardial contractility as measured on the basis of PRSW and expressed as a percentage of baseline PSRW. Protection of biventricular contractile function was similar with normothermic fibrillation and hypothermic cardioplegia.

cardiovascular physiology is advantageous because fetal myocardial function can be assessed independent of placental dysfunction. Numerous studies have demonstrated the adverse effects of fetal stress and, more specifically, fetal cardiac bypass on the placental vascular resistance, resulting in rapid cardiovascular decompensation. ${ }^{4,12}$

Interestingly, these studies revealed that normothermia during induced fibrillation afforded fetal myocardial protection equal to that provided by hypothermic cardioplegia. Hypothermia has been shown to decrease mitochondrial calcium accumulation during periods of ischemia, thereby limiting ischemia-related death. ${ }^{13}$ However, it is uncertain whether the developing myocardium will benefit from this advantage of hypothermia because of impaired myocardial calcium uptake as a result of the immature sarcolemmal structure of fetal myocytes. ${ }^{14}$ Furthermore, a cold-contrac- ture phenomenon has been observed in the immature myocardium, whereby a hypothermia-mediated inotropic effect might exacerbate ischemic injury. ${ }^{15}$ Yet even if hypothermia were to be benign or even beneficial to the fetal myocardium, it might be of limited practical use because fetal surgery will likely occur with the fetus in the uterus, severely compromising the ability to alter the temperature of the surrounding tissues.

The cardioprotective qualities of fibrillation have been extensively studied in mature myocardium. Early studies by Hottentrot and associates ${ }^{16}$ demonstrated that fibrillatory arrest caused subendocardial ischemia in adult hearts after ventricular distention. Other authors have found that fibrillation and hyperkalemic cardioplegic arrest provide similar degrees of protection of the adult myocardium. ${ }^{17,18}$ Studies in fetal lambs found that re- 


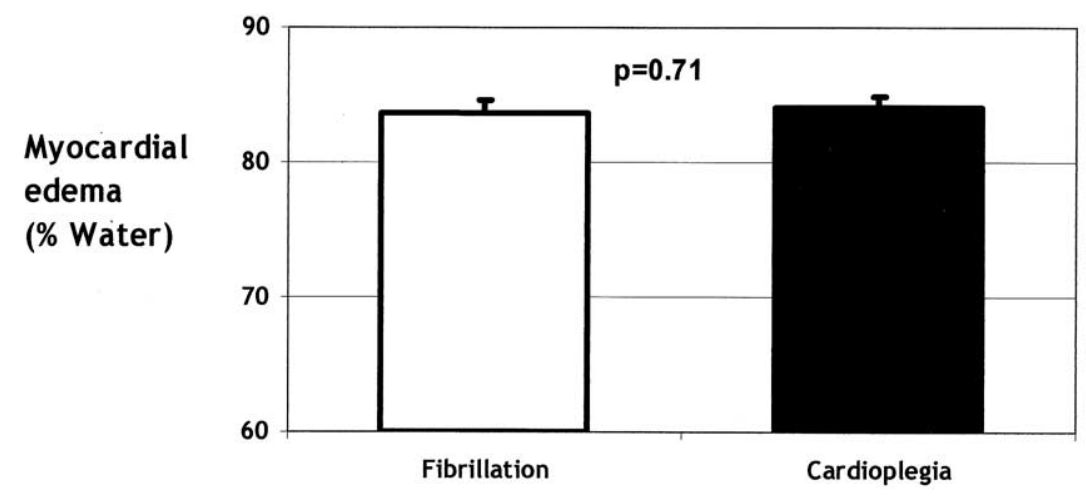

Figure 5. Myocardial water content determinations. There was no difference in postarrest myocardial edema after either fibrillation or cardioplegia. Water content with cardioplegic arrest was $83.7 \% \pm \mu 0.9 \%$ and $84.0 \mu \% \pm 1.5 \%$ $(P=.71)$.

TABLE 2. Cardioplegia composition

\begin{tabular}{cccccc}
\hline & {$[\mathrm{K}]$} & {$[\mathrm{Mg}]$} & {$[\mathrm{Ca}]$} & [Na] & $\mathbf{p H}$ \\
\hline $\mathrm{mEq}$ & 16 & 32 & 2.4 & 110 & 7.8 \\
\hline
\end{tabular}

gional myocardial blood flows were maintained during cardiac bypass with normothermic fibrillation and were markedly increased after bypass. ${ }^{6}$ This phenomenon was found to be perfusion dependent because the coronary circulation was maximally dilated. Similar mechanisms might account, in part, for the comparable levels of myocardial protection afforded by fibrillation and cardioplegia in fetal hearts.

These studies examined the effects of ischemic or fibrillatory arrest at 30 minutes. It is unclear whether similar results would be obtained at longer arrest periods. Presumably, prolonged duration of fibrillation would be less effective in protection of the fetal myocardium as a result of increased myocardial oxygen consumption. Ideally, $30 \mathrm{~min}-$ utes should be sufficient for the relatively uncomplicated fetal interventions required to correct intracardiac flow disturbances.

These results demonstrate that normothermic fibrillation provides an equal degree of fetal myocardial protection as hypothermic crystalloid cardioplegia. Fibrillation is more practical than cardioplegia in the setting of fetal cardiac surgery in that the difficulties of achieving effective hypothermia during in utero interventions are avoided. Moreover, prolonged aortic crossclamping is not required, minimizing trauma to the fragile fetal vascular tissues. Fibrillatory arrest may prove to be a valuable approach during prenatal repairs.

\section{References}

1. Rose V, Clark LE. Etiology of congenital heart disease. In: Freedom RM, Benson LN, Smallhorn JF, editors. Neonatal heart disease. New York: Springer Verlag; 1992. p. 3-13.
2. Silverman NH, Golbus MS. Echocardiographic techniques for assessing normal and abnormal fetal cardiac anatomy. J Am Coll Cardiol. 1985;5:20S-9S.

3. Fenton KM, Heinemann MK, Hanley FL. Exclusion of the placenta during fetal cardiac bypass allows improved systemic perfusion and provides important information about the mechanism of placental injury. J Thorac Cardiovasc Surg. 1993;105:502-12.

4. Sabik JF, Assad RS, Hanley FL. Prostaglandin synthesis inhibitor prevents placental dysfunction after fetal cardiac bypass. $J$ Thorac Cardiovasc Surg. 1992;103:733-42.

5. Hanley FL. Fetal cardiac surgery. Adv Cardiac Surg. 1994;5:47-74.

6. Bradley SM, Hanley FL, Duncan BW, Jennings RW, Jester JA, Verrier ED. Regional myocardial blood flow during cardiopulmonary bypass in the fetal lamb. Surg Forum. 1990;41:203-5.

7. Bradley SM, Hanley FL, Duncan BW, Jennings RW, Jester JA, Harrison MR, et al. Fetal cardiac bypass alters regional blood flows, arterial blood gases, and hemodynamics in sheep. Am J Physiol Heart Circ Physiol. 1992;263(32):H919-28.

8. Jessen ME, Abd-Elfattah AS, Wechsler AS. Neonatal myocardial oxygen consumption during ventricular fibrillation, hypothermia, and potassium arrest. Ann Thorac Surg. 1996;61:81-7.

9. Friedman WF, Pool PE, Jacobowitz D, Seagren SC, Braunwald E. Sympathetic innervation of the developing rabbit heart: biochemical and histochemical comparisons of fetal, neonatal, and adult myocardium. Circ Res. 1968;23:25-32.

10. Pegg W, Michalak M. Differentiation of sarcoplasmic reticulum during cardiac myogenesis. Am J Physiol Heart Circ Physiol. 1987;252: H22-31.

11. Gilbert RD. Effects of afterload and baroreceptors on cardiac function in fetal sheep. J Dev Physiol. 1982;4:299-302.

12. Fenton KM, Heinemann MK, Hanley FL. Exclusion of the placenta during fetal cardiac bypass allows improved systemic perfusion and provides important information about the mechanism of placental injury. J Thorac Cardiovasc Surg. 1993;105:502-12.

13. Buja LM, Chien KR, Burton KP. Membrane damage in ischemia. $A d v$ Exp Med Biol. 1982;161:421-31.

14. Mahoney L. Maturation of calcium transport in cardiac sarcoplasmic reticulum. Pediatr Res. 1988;24:639-43.

15. Rebeyka IM, Hanan SA, Borges MR. Rapid cooling contracture of the myocardium. J Thorac Cardiovasc Surg. 1990;100:240-9.

16. Hottentrot CE, Buckberg GD. Studies of the effects of ventricular fibrillation on the adequacy of regional myocardial flow. $J$ Thorac Cardiovasc Surg. 1974;68:626-33.

17. Bessho R, Chambers DJ. Experimental study of intermittent crossclamping with fibrillation and myocardial protection: reduced injury from shorter cumulative ischemia or intrinsic protective effect? $J$ Thorac Cardiovasc Surg. 2000;120:528-37.

18. Grotte GJ, Levine FH, Kay HR, Fallon JT, Austen WG, Buckley MJ. 
Effect of ventricular fibrillation and potassium induced arrest on myocardial recovery in hypothermic hearts. Surg Forum. 1980;31:296-8.

\section{Discussion}

Dr Davis C. Drinkwater (Nashville, Tenn). I very much enjoyed the article. One point and question come to mind with this potential technique. Myocardial distention on a ventricle might be injurious, particularly in an immature heart under fibrillatory arrest. Therefore, if you have a left-sided valve problem, presumably you are going to try to open that valve. Are you concerned about creating an insufficient valve under those conditions as opposed to the crossclamp condition in terms of less precise pressure control and causing left ventricular distention with possible poor myocardial protection?

Dr Malhotra. You are always worried about distention when using fibrillation, but if you can restore the patterns of blood flow, you can avoid the potentially adverse effects of hypothermia. From a practical standpoint, fibrillation is easier to accomplish in the fetus than hypothermic cardioplegic arrest.

Dr John E. Mayer, Jr (Boston, Mass). I am sure this is not an easy model to work with, and it has got to be a real challenge to get these things to work. There are a few interesting questions that sort of came up in my own mind, one of which is with the fetal $\mathrm{PO}_{2}$ values being quite a bit lower than they are in postnatal life, how do you think that the effect of using a Langendorf preparation, where you almost certainly have hyperoxic conditions, might yield results that would be at variance with what you might see in a fetal myocardium?

Dr Malhotra. That is excellent point. We actually did take that into account, and we were very sensitive to the effects of hyperoxia. We did, at periodic intervals, perform blood gas analysis of the perfusate and attempted to keep the $\mathrm{Po}_{2}$ between 30 and $40 \mathrm{~mm}$ $\mathrm{Hg}$.

Dr Mayer. Okay. And then the second issue, which is related: have you considered using a blood prime or a blood perfusate in your circuit rather than the crystalloid Langendorf type preparation?
Dr Malhotra. Actually, when we first thought about designing the model, we wanted to use a blood prime, but, as you know, the difficulty was in having enough fetal blood to use. Because of the differences in fetal and adult hemoglobin, we did not want to use pooled blood from adults.

Dr Frank W. Sellke (Boston, Mass). I agree, this is an outstanding study; it is extremely novel and innovative. You only gave one dose of cardioplegia crystalloid solution. Do you think that provided optimal protection?

How could you modify the cardioplegic solution to take into consideration the immature nature of the calcium-handling system within the immature myocardium to provide optimal protection?

Dr Malhotra. Repeat your first question, please.

Dr Sellke. You gave one dose of the crystalloid cardioplegia.

Dr Malhotra. Right. That is one of the things we want to look at in the future. Dosing of cardioplegia in neonates varies from institution to institution, but from discussions with the perfusionists and the staff at the University of California, we were comfortable with using one dose for a 30-minute arrest period. Of course, in the future, we can look at multiple dosing. In terms of modifying cardioplegia to account for the immaturity of fetal calcium regulation, we presented our studies at the American Heart meeting this past November, in which we altered the calcium concentration in the cardioplegic solutions, and found that there is a profound influence of calcium cardioplegic concentration.

Dr Erik A. Beyer (Cleveland, Ohio). I had a question about the Langendorf model. You seem to suspend the heart from the aorta and the pulmonary artery, is that correct?

Dr Malhotra. From the SVC and the aorta.

Dr Beyer. Does that have any effect on the hemodynamic measurements that you have? Did you try to rest the heart from its apex or posteriorly from the atrium to more simulate a more normal situation?

Dr Malhotra. We went through a number of iterations with this model, and initially we had started out with suspending or having it rest on a platform, but we graduated to this and did not find any difference in hemodynamic performance. 